Erschienen in: Bausch, Karl-Heinz/Grosse, Siegfried (Hrsg.): Spracherwerb und Sprachunterricht

für Gehörlose. Zielsetzungen und Probleme. - Tübingen: Niemeyer, 1989. S. 1-8.

(Reihe Germanistische Linguistik, 94)

Karl-Heinz Bausch/Siegfried Grosse

\title{
Zur Einführung: SPRACHUNTERRICHT FÜR GEHÖRLOSE UND LINGUISTIK
}

In der Bundesrepublik gibt es schätzungsweise 600000 stark Hörgeschädigte oder Gehörlose.1) Sie können Sprache nur über visualisierte Formen (Schrift, Ablesen vom Munde, Fingeralphabet, Gebärdensprache) wahrnehmen, das heißt: Von Geburt an oder in der frühen Jugend Ertaubte können die Sprache nicht in natürlichen Interaktionssituationen ungesteuert erwerben, sondern bedürfen spezifischer sprachpädagogischer Förderung. Auch spät Ertaubte sind auf sprachpädagogische Förderung angewiesen, um weiterhin an der mündlichen Kommunikation teilhaben zu können und keinen Sprachverlust zu erleiden.

Die Beiträge des vorliegenden Bandes sind aus einem Kolloquium hervorgegangen, zu dem die Kommission für Fragen der Sprachentwicklung des Instituts für deutsche Sprache zum 26. und 27. Juni 1987 eingeladen hatte. Warum die Sprache Gehörloser zum Gegenstand der Tagung gewählt wurde, bedarf einer Erläuterung: Linguisten beschäftigen sich in der Regel mit dem Normalfall, d.h. mit der Sprache vollsinniger Sprecher, die Sprache über das Gehör wahrnehmen und mündlich artikulieren können. Die Bindung an den Laut gehört in der Disziplin geradezu zum definitorischen Bestandteil von Sprache.2) Wenn therapeutische Aspekte ins Blickfeld geraten, stehen die Untersuchung sozialisations- oder physisch/psychisch bedingter Abweichungen im Vordergrund, die den pädagogischen Problemfällen der Regelschulen oder dem Bereich der Erwachsenenrehabilitation zuzuordnen sind (dialektale Unterschiede, Pidginisierungen, Analphabetismus, Legasthenie, Aphasie). Nicht in diesen Rahmen passende Fragestellungen weist man hierzulande der Sonderpädagogik oder Psychologie zu, also auch das Kommunikations- und Sprachverhalten Gehörloser. Für die etablierte Linguistik scheint das Thema weder von lebenspraktischem noch von theoretischem Interesse zu sein.

Mit dem Kolloquim wollten wir das Interesse für Abweichungen vom Normalkanon wecken; denn das Kommunikations- und Sprachverhalten der Gehörlosen zeigt schnell einige Aspekte, die in anderen Bereichen der Linguistik von Bedeutung sind:

- Zwischen dem Spracherwerb (der Sprachanbildung) Gehörloser und dem Zweitspracherwerb Hörender gibt es - trotz aller Unterschiedlichkeit der Ausgangsposition - Parallelen, die aus linguistischer Sicht einer eingehenden Betrachtung bedürfen. Hier seien nur einige Stichworte erwähnt: situativ orientiertes Lernen, Auswahl grammatischer Strukturen, Grundwortschatz, Generalisierungs- und Simplifizierungsstrategien der Lerner. Diese Fragen gehören zu den zentralen Gegenstandsbereichen der Sprachlehr- und Sprachkontaktforschung. 3)

- In den letzten Jahren ist in der Gehörlosenpädagogik eine Diskussion um den Status der Gebärdensprache im Unterricht entstanden. Dabei geht es einerseits um die Frage der Funktion der Gebärdensprache als Ausgangssprache bzw. Verdeutlichungshilfe für den Sprach- und Sachunterricht und um ihre 
semantische und syntaktische Genauigkeit bezogen auf das Deutsche, andererseits aber auch um die Absicht, die Gebärdensprache zu standardisieren und damit ihren Gebrauch zu vereinheitlichen.4) Dahinter steht letztlich die Frage nach strukturellen Ähnlichkeiten zwischen zwei semiotischen Systemen mit medial unterschiedlichen Darstellungsformen.

- Seit den siebziger Jahren beschäftigen sich besonders in den USA Linguisten und Psychologen intensiv mit der Beschreibung der amerikanischen Gebärdensprache (American Sign Language, kurz: ASL). Sie sind zu dem Ergebnis gekommen, daß die ASL in Struktur und Leistungsfähigkeit eine voll ausgebaute Sprache ist, die dem Englischen nicht nachsteht. Aufgrund dieser Ergebnisse neigen einige Linguisten dazu, die traditionelle Definition, Sprache sei an lautliche Präsentation gebunden, in Frage zu stellen.5)

- Die Frage nach dem Ursprung der Sprache und die Entwicklung von Kultursprachen wurde in den letzten Jahren von seiten der kognitiven Psychologie/Linguistik und der Creoleforschung mit empirischen Argumenten wieder aufgegriffen. Auch hier können weitere Untersuchungen zur Gebärdenkommunikation aufschlußreich sein.6)

- Im Zusammenhang mit der kognitiven Entwicklung Gehörloser wurde von Sprachpsychologen die alte Frage nach der Beziehung zwischen Sprache und Denken wieder aufgerollt, die lange Zeit eine Domäne der Sprachphilosophie war. Die Ergebnisse stellen traditionelle Denkmuster unter Linguisten in Frage.7)

- In den letzten Jahren beschäftigen sich Linguisten und Psychologen stärker mit der Beziehung zwischen Schrift und Laut und mit Erwerb und Funktion der Schriftsprache. Da für Gehörlose die Schrift das zentrale Medium für den Spracherwerb ist, sind wertvolle Anregungen auch von Gehörlosenpädagogen zu erhoffen.8)

Theoretische Aspekte der Kommunikation Gehörloser wurden aus biologischer und psychologischer Sicht auf einer Tagung 1980 eingehend behandelt.9) Ziel des Mannheimer Kolloquiums sollte dagegen sein, von der sprachpädagogischen Praxis ausgehende linguistische Fragen in den Vordergrund zu stellen und ihre theoretischen Implikationen aufzunehmen.

Medizinische Forschungsergebnisse und therapeutische Folgerungen erörtert P. Plath: Die Ausblidung der Hörfähigkeit kann heute bereits pränatal oder in den ersten Lebensmonaten diagnostiziert werden. Eine sinnvolle Therapie von Hörschädigungen kann deshalb schon in den ersten Lebensjahren einsetzen. Neuere Forschungsergebnisse legen nahe, dabei auch die Aktivierung der auditiven Sinnesreize einzubeziehen; denn der vorhandene Rest an Hörfähigkeit bedarf einer Stimulierung, um sich weiter entwickeln zu können.

Soziologischen Aspekten der Gehörlosigkeit wendet sich H. Ding zu: Sie ist eine unsichtbare Schädigung, die sich erst in der Kommunikation dadurch als Störung auswirkt, daß Gehörlose nur bedingt in der Lage sind, klare Beziehungsangebote zu machen und zu verstehen. 
Der Hörende reagiert darauf mit schockartigem Verhalten und Reduktion kommunikativer Angebote. Als Folge zieht sich der Gehörlose unter seinesgleichen in die Gehörlosengemeinschaft zurück. Unter diesen Gegebenheiten stellt sich die Frage nach der Art sozialer Eingliederungskonzepte: Soll eine sprachliche Anpassung an den Standard der Gemeinschaft der Hörenden durch intensiven Sprachunterricht im Vordergrund stehen, soll die Integration in die Gehör losengemeinschaft durch Förderung der Gebärdensprache betont werden oder gibt es einen Mittelweg?

Den Lernzielkatalog und dessen Umsetzung im Sprachunterricht für Gehörlose erörtert H. Jussen: Zentrale Aufgabe an den Schulen ist die polysensorische Einübung der Lautsprache durch vibrotaktile und optische Verfahren, die den Erwerb einer annähernd korrekten Sprachartikulation ermöglichen soll. Dazu gehört auch das Entschlüsseln von Äußerungen an den Absehgestalten des Mundes beherrschen zu lernen. Die fehlende soziale Erfahrung Gehörloser wird durch interaktionale Spracherziehung kompensiert. Der Erwerb semantisch-pragmatischer Strukturen erfolgt über die Vermittlung prototypischer Lebensstrukturen. So entsteht ein Gerüst von kognitiven und sprachlichen Grundstrukturen, das durch Analogieverfahren erweitert werden kann. Bisher noch unbefriedigend gelöst sind die Vermittlung sprachlicher Routinen der Gesprächsführung und des Rollenverhaltens sowie die Strukturbeziehungen zwischen Gebärdensprache und Lautsprache. Zur Lösung dieser Aufgaben seien auch Linguisten aufgefordert.

Einen Überblick über Methoden des Lautspracherwerbs und den Einsatz von Gebärden im Unterricht gibt 0 . Kröhnert: Aus dem lautsprachlich akzentuierten Bildungsauftrag der Gehörlosenschulen heraus wurden besonders in den letzten Jahrzehnten unterschiedliche technische Methoden entwickelt, die unter Nutzung polysensorischer Verfahren den Lautspracherwerb stützen. Die Gebärde bleibt nach wie vor nur eines der Vehikel zum Erwerb der Lautsprache und ist deshalb auf das Handalphabet und lautsprachlich orientierte Gebärden beschränkt. Versuche, das Prinzip der Lautsprachpriorität anzutasten, hat es immer wieder gegeben. In Europa konnten sie sich jedoch aus Überzeugung vieler Pädagogen heraus, extensive Verwendung von Gebärden hemmen den Lautspracherwerb, nicht durchsetzen.

Unterschiedliche Aspekte der Gebärdensprache, die erst in den letzten Jahren wieder stärkere Beachtung gefunden hat, sprechen die drei darauffolgenden Beiträge an.

Die Gebärdensprache als Erstsprache Gehörloser und deren Status als Sprachsystem stellt S. Prillwitz in den Vordergrund. Langzeituntersuchungen in Gehörlosenfamilien haben gezeigt, daß gehörlose Kinder von ihren Eltern ein Repertoire an Gebärden für die Alltagskommunikation erwerben, das analog zur Lautsprache mit einer endlichen Zahl von Regeln auf unterschiedlichen Ebenen beschrieben werden kann. Diese Gebärdensprache ist allerdings nicht voll standardisiert, weil sie bewußt aus dem pädagogischen Bereich herausgehalten wurde. Er regt an, die Gebärdensprache im Sach- und Sprachunterricht intensiver als bisher einzusetzen, weil sie bei Gehörlosen gleichsam die Stelle der Erstsprache bei Hörenden einnimmt. 
Ausgehend von Überlegungen der Arbeitsgemeinschaft Gebärdenlexikon (s. Maisch/Wisch 1987) diskutiert G. Rammel Probleme des lautsprachbegleitenden Gebärdenverfahrens, das auf eine strukturelle Angleichung von Lautsprache und Gebärdensprache hinzielt. Noch nicht entschieden sei z.B. die Frage, inwieweit man Gebärden für Oberflächenstrukturen des Deutschen (z.B. Plural, Flexion, Modus, Passiv, Wortarten) in die Gebärdensprache einführen sollte. Eine solche struktruelle Angleichung berge die Gefahr der grammatischen Überzeichnung der Gebärdensprache. dahinter steht die Frage, inwieweit eine Gebärdensprache den Strukturen einer Lautsprache folgen kann, ohne ihre Funktion als Kommunikationsmittel unter Gehörlosen zu verlieren.

Überlegungen zu Zielgruppe, Aufbau und Benutzerfreundlichkeit von Gebärdenlexika anhand der für den deutschen Sprachraum verfügbaren Gebärdenlexika (Maisch/Wisch 1987; Starcke/Maisch 1977; Gehörlosenund Schwerhörigenverband der DDR 1985) stellt K.-B. Günther an: Benutzer seien in erster Linie Eltern gehörloser Kinder, Lehrer und Sozialarbeiter, d.h. Hörende, die ein solches Lexikon als Lehrbuch und Nachschlagewerk (Handbuch) benutzen. Zentral ist die Frage nach der Auswahl der Einträge (u.a. Grund(wort)gebärdenschatz, Internationalismen, Fremd- und Lehn(wörter)gebärden) und das Ordnungsprinzip, nach dem die Einträge erfolgen. Das von einer alphabetischen Abfolge abweichende, besonders in Maisch/Wisch (1987) eingeschlagene Verfahren erschwere die Zugriffsmöglichkeiten für den Benutzer.

Unterschiedliche Aspekte der Sprachvermittlung und Sprachbeschreibung sprechen die vier darauffolgenden Beiträge an.

Den Doppelaspekt von Schriftbildern im Unterricht betont B. Fischer. Sie dienen als Träger von Inhalten einerseits dem eigentlichen Sprachaufbau auf grammatischer und semantischer Ebene. Andererseits erfüllen sie aber auch eine zentrale Führungsfunktion für den Erwerb einer möglichst exakten Artikulation. Die beiden Aspekte sollten auch durch unterschiedliche Unterrichtsmaterialien und Lernschritte auseinandergehalten werden. Dadurch könnte erreicht werden, daß die Schüler eine überschaubare Anzahl von Sprachelementen wahrnehmen und reproduzieren lernen, ohne allzu großen lesetechnischen Mißerfolgen ausgesetzt zu sein.

Fragen der Auswahl, Vereinfachung und Abfolge von Sprachstrukturen des Deutschen im Gehörlosenunterricht, die an vergleichbare Probleme im Unterricht Deutsch als Fremdsprache erinnern, schneidet J. Tigges an. Er nennt einige im Sprachunterricht für Gehörlose etablierte Grundbestände von $z u$ vermittelnden Strukturen auf morphophonologischer, syntaktischer und lexikalisch-semantischer Ebene.

Möglichkeiten des computergestützten interaktiven Lernens im fachorientierten Sprachunterricht zeigt K.-H. Wisotzki auf: Aufgrund einer Fehleranalyse von Schülertexten aus dem Physik- und Chemieunterricht der Sekundarstufe wurde ein Lernprogramm entwickelt, das schrittweise an fachtexttypische syntaktische Strukturen heranführt. In dem Zusammenhang wird die Frage nach der Anwendbarkeit bzw. Eignung von unterschiedlichen Grammatikmodellen für Sprachpro- 
gramme angeschnitten. Bessere Lernerfolge seien insbesondere deshalb zu erwarten, weil computergestützte interaktive Lernprogramme eine frei wählbare übungszeit mit individuellen Einstiegsmöglichkeiten ermöglichen, die nicht an die Unterrichtssituation gebunden sein müssen. Dadurch könne der Sprachumsatz, die Durchsichtigkeit des Lernprozesses und die Erfolgskontrolle für den Lernenden selbst gesteigert werden.

Einen Einblick in die Grundlagenforschung zur Sprachkompetenz Gehörloser gibt K. Schulte. An der Forschungsstelle der Pädagogischen Hochschule Heidelberg für angewandte Sprachwissenschaft zur Rehabilitation Behinderter wird an erhobenem Sprachmaterial die Sprachkompetenz unterschiedlicher Gruppen untersucht und aus hörbehinderungsspezifischen Abweichungen Konsequenzen für die Sprachanbildung auf verschiedenen Sprachentwicklungsstufen abgeleitet. Der Beitrag schneidet auch einige Analyse- und Beschreibungsprobleme im Bereich der Bedeutungsrekonstruktion, syntaktischen Segmentierung und grammatischen Kategorisierung an, die ähnlich gelagert auch aus der Analyse von Materialien aus dem Zweitspracherwerb und von Pidginsprachen bekannt sind.

Wir hoffen, daß die Beiträge des Bandes einen Einblick in aktuelle linguistische Fragestellungen zum Sprachunterricht Gehörloser aus der Sicht der Gehörlosenpädagogik vermitteln. Es schien uns sinnvoll, diese Bestandsaufnahme zu machen, um auf einen Forschungsbereich hinzuweisen, um den sich Linguisten intensiver kümmern sollten.

\section{Anmerkungen}

1. Genaue statistische Angaben gibt es nicht. Krüger in Jussen/Kröhnert (1982) $38 \mathrm{ff}$. rechnet Erhebungen aus den USA auf die Bundesrepublik um. Danach sind $5 \%$ der Bevölkerung (ca. 3.000.000) "signifikant bilateral hörgestört". $1 \%$ der Bevölkerung (ca. 600.000) kann die "Umgangssprache nicht hören oder verstehen", darunter sind schätzungsweise 50.000 Gehörlose. 1975 besuchten 5.215 "praktisch taube" Kinder im Alter zwischen 6 und 15 Jahren die Schule.

2. Die Position findet sich schon 1951 bei $F$. de Saussure (1969) 45:

"Langue et écriture sont deux systèmes des signes distincts; l'unique raison d'être du second est de représenter le premier; l'object lingustique n'est pas défini par la combinaison du mot écrit et du mot parlé; ce dernier constitue à lui seul cet objet".

3. Zur Verdeutlichung der unterschiedlichen Interessen und gemeinsamen Berührungspunkte s.z.B. Ferguson (1971); Bickerton (1977) und Corder/Roulet (1977). Zu analogen Strukturen von Bedeutungskonstitution und Spracherwerb in der Gebärdenkommunikation und der Kommunikation mit 'simplifizierten' Sprachformen, s. Bausch (1989).

4. Seit dem Mailänder Kongreß 1880 steht die Vermittlung der Laut- 
sprache im Vordergrund. der Status der Gebärden im Unterricht wurde zuletzt 1982 im sogenannten Münchner Gebärdenpapier festgeschrieben (s. Bundesarbeitsgemeinschaft der Elternvertreter, 1982). Zur Standardisierung der Gebärdensprache s. die Einleitung in Maisch/Wisch (1987).

5. Eine Beschreibung der ASL von der Morphologie über Syntax und Wortbildung bis hin zur poetischen Kreativität geben Klima/ Bellugi (1979). Die Arbeit - ein Ergebnis mehrjähriger Forschungen am Salk Institute for Biological Studies - führt ein drucksvoll die Leistungsfähigkeit der ASL vor. Einen guten Überblick über die unterschiedlichen Gebärdensysteme gibt Rammel (1981). Eine Übersicht über allgemeine Entwicklungsprinzipien visueller Kommunikation gibt Kugler-Kruse (1988).

6. S. z.B. das anregende und provozierende Buch von Bickerton (1981).

7. s. besonders Furths von der Piaget-Schule ausgehende Untersuchungen (Furth 1972).

8. Zur Schriftlichkeit s. z.B. die von Coulmas/Ehlich (1983), Grosse (1983) und Günther/Günther (1983) herausgegebenen Sammelbände. Speziell zur Schriftsprache bei Hör- und Sprachgeschädigten s. Günter (1985). Zu Alltagsformen des Schreibens s. Buhofer (1985).

9. In einer der Dahlem Konferenzen in Berlin. Die Beiträge wurden herausgegeben von Bellugi/Studdert-Kennedy (1980).

\section{Literatur}

Bausch, K.-H. (1989): Kommunikative Kompetenz, Grammatik und Sprachnorm - Einige Bemerkungen am Beispiel der Gebärdensprache und anderer 'simplifizierter' Sprachformen. In: Rickheit, G./Wichter, S. (Hrg.) (1989): Dialog. Tübingen.

Bellugi, U./Studdert-Kennedy, M. (Hrsg.) (1980): Signed and Spoken Language. Biological Constraints on Linguistic Form. Weinheim.

Bickerton, D. (1977): Pidginization and Creolization: Language Acquisition and Language Universals. In: Valdman, A. (Hrsg.) (1977): Pidgin and Creole Linguistics. Bloomington, London. 49-69.

Bickerton, D. (1981): Roots of Language, Ann Arbor.

Buhofer, A.H. (1985): Schriftlichkeit im Alltag. Theoretische und empirische Aspekte am Beispiel eines schweizer Industriebetriebs (Züricher germanistische Studien, Band 2) Bern. 
Bundesarbeitsgemeinschaft der Elternvertreter und Förderer Deutscher Gehörlosenschulen e.V. (Hrg.) (1982): Kommunikation mit Gehörlosen in Lautsprache und Gebärde. München.

Corder, S.P./Roulet, E. (Hrg.) (1977): The Notions of Simplification, Interlanguages and Pidgins and their Relation to Second Language Pedagogy. Geneve.

Coulmas, F./Ehlich, K. (Hrg.) (1983): Writing in Focus. The Hague/Paris.

Ferguson, Ch. A. (1971): Absence of Copula and the Notion of Simplicity: A Study of Normal Speech, Baby Talk, Foreigner Talk, and Pidgins. In: Dil, A.S. (Hrg.) (1971): Language Structure and Language Use. Essays by Charles A. Ferguson. Stanford. 277-292.

Furth, H.G. (1972): Denkprozesse ohne Sprache. Düsseldorf. (Original: Thinking without Language, London 1966).

Gehörlosen- und Schwerhörigenverband der DDR (Hrg.) (1985): Lehr- und Übungsbuch der Gebärden Gehörloser. Berlin (DDR).

Grosse, S. (Hrg.) (1983): Schriftsprachlichkeit (Sprache der Gegenwart. Band 59). Düsseldorf.

Günther, K.B. (1982): Schriftsprache bei Hör- und sprachgeschädigten Kindern (Hörgeschädigtenpädagogik. Beiheft 9). Heidelberg.

Günther, K.B./Günther, H. (Hrg.) (1983): Schrift, Schreiben, Schriftlichkeit (Reihe Germanist. Linguistik, Band 49). Tübingen.

Jussen, H./Kröhnert, O. (Hrg.) (1982): Pädagogik der Gehörlosen und Schwerhörigen. Handbuch der Sonderpädagogik. Band 3. Berlin.

Klima, E.S./Bellugi, U. (1979): The Signs of Language. Cambridge, Mass./London.

Kugler-Kruse, M. (1988): Die Entwicklung visueller Zeichensysteme. Von der Geste zur Gebärdensprache (Bochumer Beiträge zur Semiotik. Band 20) Bochum.

Maisch, G./Wisch, F.H. (1987): Gebärdenlexikon. Band 1: Grundgebärden. Hamburg.

Rammel, G. (1981): Untersuchungen zur Zeichensystematik der Gebärden und der Gebärdensprachen. (Hörgeschädigtenpädagogik. Beiheft 7.) Heidelberg.

Saussure, F. de (1969): Cours de Linguistique Generale. 3. Aufl. Paris. 
Starcke, H./Maisch, G. (1977): Die Gebärden der Gehörlosen. Ein Hand-, Lehr-, und Übungsbuch (hrsg. von der Deutschen Gesellschaft zur Förderung der Hör- und Sprachgeschädigten). Hamburg. 